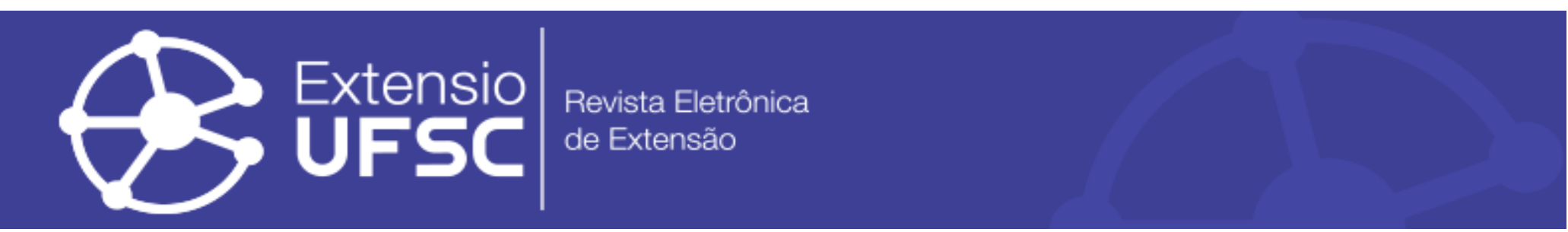

\title{
GRUPO DE GESTANTES E CASAIS GRÁVIDOS: EDUCAÇÃO EM SAÚDE EM TEMPOS DE PANDEMIA
}

\author{
Margarete Maria de Lima \\ Universidade Federal de Santa Catarina \\ margarete.lima@ufsc.br
}

Maria Eduarda de Pinho Aranha

Universidade Federal de Santa Catarina

mariaeduardapinho75@gmail.com

Clara de Andrade Leal

Universidade Federal de Santa Catarina clara13.andrade@gmail.com
Roberta Costa

Universidade Federal de Santa Catarina roberta.costa@ufsc.br

Maria de Fátima Motta Zampieri Universidade Federal de Santa Catarina fatimazampieri@gmail.com

Zaira de Oliveira Custódio

Universidade Federal de Santa Catarina zairacustodiohu@gmail.com

\section{Resumo}

O presente estudo tem como objetivo relatar a experiência do desenvolvimento de atividades educativas virtuais no Grupo de Gestantes e Casais Grávidos da Universidade Federal de Santa Catarina diante da pandemia de Covid-19. O Grupo de Gestantes e Casais Grávidos é uma atividade educativa de extensão, articulada ao ensino e à pesquisa, de abordagem interdisciplinar, dirigida a gestantes e seus acompanhantes. Diante das recomendações de distanciamento social, a coordenação do projeto decidiu suspender as suas atividades no formato presencial, realizando-as por meio de encontros sistemáticos online, que ocorreram entre os meses de março e julho de 2020. A execução destes encontros facilitou o acesso a informações de qualidade, possibilitou a formação de uma rede de apoio entre os participantes, proporcionou um local para exposição de dúvidas, dificuldades e inseguranças enfrentadas pela vivência da gestação e parto durante a pandemia, mantendo, deste modo, ativa a contribuição do projeto à comunidade. O processo de adaptação das atividades do grupo de gestantes à modalidade online representou para as discentes uma oportunidade de conhecer novas maneiras de colocar em prática a promoção da saúde, compartilhar conhecimento e trocar experiências, mesmo que a distância. Esta experiência mostrou que é possível e necessário inovar a extensão universitária diante do desafio imposto pela pandemia da Covid-19.

Palavras-chave: Educação em Saúde. Gravidez. Infecções por Coronavirus. Extensão Comunitária.

\section{GROUP OF PREGNANT WOMEN AND PREGNANT COUPLES: HEALTH EDUCATION IN PANDEMIC TIMES}

\begin{abstract}
This study aims to report the experience of the development of virtual educational activities in the Group of Pregnant Women and Pregnant Couples of the Federal University of Santa Catarina during the Covid-19 pandemic.The Group of Pregnant Women and Pregnant Couples is a educational extension activity, linked to teaching and research, interdisciplinary approach, aimed at pregnant women and their companions. In view of the recommendations for social isolation, the project's coordination decided to suspend its activities in person, carrying out them through systematic online meetings, which took place between the months of March and July 2020. The execution of these meetings enabled the formation of a support network among the participants, and facilitated access to quality information, providing a place for exposing insecurities, doubts and difficulties faced by experiencing a pregnancy and childbirth during the pandemic, thus maintaining the project's contribution to the community. The process of adapting the activities of the group of pregnant women to the online modality represented an opportunity for students to discover new ways of putting health promotion into practice, sharing knowledge and exchanging experiences, even at a distance. This experience revealed that it is possible and necessary to innovate university extension in the face of the challenge imposed by the Covid-19 pandemic.
\end{abstract}

Keywords: Health Education. Pregnancy. Coronavirus Infections. Community-Institutional Relations 
Grupo de gestantes e casais grávidos: educação em saúde em tempos de pandemia

\title{
GRUPO DE GESTANTES E PAREJAS EMBARAZADAS: EDUCACIÓN PARA LA SALUD EN TIEMPOS DE PANDEMIA
}

\begin{abstract}
Resumen
Este estudio tiene como objetivo reportar la experiencia de desarrollar actividades educativas virtuales en el Grupo de Mujeres Embarazadas y Parejas Embarazadas de la Universidad Federal de Santa Catarina ante la pandemia del Covid-19. El Grupo de Mujeres Embarazadas y Parejas Embarazadas es una actividad de extensión educativa, ligada a la docencia y la investigación, de enfoque interdisciplinario, dirigida a mujeres embarazadas y sus acompañantes. Ante las recomendaciones para el aislamiento social, la coordinación del proyecto decidió suspender sus actividades de manera presencial, llevándolas a cabo a través de reuniones sistemáticas en línea, que tuvieron lugar entre los meses de marzo y julio de 2020. La ejecución de estas reuniones permitió la formación de una red de apoyo entre las participantes, facilitó el acceso a información de calidad, y brindó un espacio para exponer las inseguridades, dudas y dificultades que enfrenta la experiencia del embarazo y parto durante la pandemia, manteniendo, así la contribución del proyecto a la comunidad. El proceso de adecuación de las actividades del grupo de gestantes a la modalidad online representó una oportunidad para que los estudiantes descubrieran nuevas formas de poner en práctica la promoción de la salud, compartiendo conocimientos e intercambiando experiencias, incluso a distancia. Esta experiencia reveló que es posible y necesario innovar la extensión universitaria ante el desafío que impone la pandemia Covid-19.
\end{abstract}

Palavras clave: Educacíon en Salud. Embarazo. Infecciones por Coronavirus. Relaciones Comunidad-Institución. 
Grupo de gestantes e casais grávidos: educação em saúde em tempos de pandemia

\section{INTRODUÇÃO}

No final do ano de 2019, a Organização Mundial de Saúde (OMS) emitiu um alerta epidemiológico sobre um tipo de pneumonia de etiologia desconhecida que acometeu uma quantidade significativa de pessoas na cidade de Wuhan, na China. Em janeiro de 2020, pesquisadores chineses identificaram o SARS-CoV-2 (Severe acute respiratory syndrome coronavirus 2) como agente etiológico da doença respiratória que foi denominada Covid-19, a qual se espalhou rapidamente pelo mundo (ARAÚJO, OLIVEIRA, FREITAS, 2020). Perante o cenário mundial, diversos países elaboraram uma série de ações para diminuir a transmissão do vírus. As medidas compreendem o isolamento de casos positivos, o uso de máscaras faciais e medidas de distanciamento social (AQUINO, 2020).

No campo dos cuidados em saúde, a situação de pandemia e a recomendação de distanciamento social trazem a necessidade de novas medidas para a garantia do cuidado em todos os níveis, exigindo a reorganização e a reformulação de condutas e práticas, tanto no âmbito do atendimento à saúde como no da educação em saúde. A educação em saúde tem como principal objetivo o compartilhamento de conhecimento sobre saúde, visando à conscientização da comunidade sobre um tema específico. No contexto da pandemia, ações educativas em saúde contribuem com a propagação de informações em relação a medidas de prevenção e cuidado pessoal, buscando reduzir a disseminação da Covid-19 (KRAMERI et al., 2020). Neste sentido, considerando que as atividades educativas realizadas em grupos foram suspensas a partir da recomendação de distanciamento social, surgiu a necessidade de se repensar e reformular essas práticas, para atender as demandas da sociedade.

No que se refere à educação em saúde no cuidado à mulher no ciclo gravídico-puerperal, os grupos de gestantes são estratégias para o enfrentamento das mudanças decorrentes da gestação, uma vez que possuem um cunho terapêutico e informativo para gestantes e acompanhantes. Estes grupos possibilitam uma espécie de filtro de práticas, onde, através de conversas e discussões, se exerce uma troca de conhecimentos ou experiências (NUNES, 2017). Em um contexto adverso como o da pandemia da Covid-19, esse espaço de educação em saúde desenvolvido por meio de recursos tecnológicos se torna um mecanismo de suporte à mulher e seu acompanhante durante a gravidez e no preparo ao parto e nascimento.

Desenvolver a assistência a gestantes em contexto de incertezas e pouco consenso entre a comunidade científica sobre a gravidade da Covid-19 durante a gravidez se mostra como um desafio para os profissionais de saúde que precisam repensar suas práticas, para amenizar ou 
Grupo de gestantes e casais grávidos: educação em saúde em tempos de pandemia

impedir os impactos da doença para o binômio mãe-bebê, bem como pensar em estratégias de cuidado que acolham as demandas das mulheres da gravidez ao período pós-parto e assegurem suporte social e institucional adequado para este momento tão delicado na vida das futuras mães (ESTRELA et al, 2020).

Com o intuito de compartilhar conhecimentos sobre a gestação, parto e nascimento, e possibilitar troca de experiências e criação de vínculo entre gestantes e casais grávidos, foi criado em 1996 o Grupo de Gestantes e Casais Grávidos (GGCG), da Universidade Federal de Santa Catarina (UFSC). O Grupo é uma atividade educativa de extensão articulada ao ensino e à pesquisa. As atividades de extensão são consideradas um processo interdisciplinar, político educacional, cultural, científico e tecnológico, que promove interação entre as instituições de ensino superior e os outros setores da sociedade, por meio da produção e da aplicação do conhecimento (BRASIL, 2018). Neste sentido, as atividades do GGCG possuem como base principal a articulação junto à comunidade, promovendo um espaço de troca de informações entre gestantes, acompanhantes, familiares e profissionais da área da saúde.

Em março de 2020, as atividades presenciais foram suspensas na UFSC, visando atender às recomendações dos órgãos governamentais para reduzir a disseminação do vírus SARS-CoV-2 na população e dentro da comunidade universitária (UNIVERSIDADE FEDERAL DE SANTA CATARINA, 2020). Esta medida afetou todas as atividades de ensino, pesquisa e extensão, de graduação e pós-graduação. A partir da liberação da nota oficial por parte da administração central da Universidade, e considerando a importância das medidas de distanciamento social, principalmente para indivíduos dos grupos de risco, a coordenação do GGCG decidiu dar continuidade às atividades do projeto por meio de encontros sistemáticos online.

A tecnologia e os ambientes virtuais são espaços que representam uma democratização do ensino e um crescimento das oportunidades para aprender. Neles, as atividades de ensino deixam de ser delimitadas geograficamente e condicionadas a um lugar, possibilitando que pessoas de diversos locais, cidades e até países se reúnam em um ambiente virtual para trocar experiências e informações. A realização de atividades online cria a possibilidade de mudanças no processo de ensino-aprendizagem, proporcionando um espaço de interação entre os inscritos (GONÇALVES, 2011).

No contexto da pandemia da Covid-19, as atividades de ensino-aprendizagem realizadas de maneira virtual vêm sendo muito utilizadas como estratégia para enfrentar o distanciamento social e atender os decretos governamentais. Deste modo, o presente estudo tem por objetivo relatar a 
Grupo de gestantes e casais grávidos: educação em saúde em tempos de pandemia

experiência de atividades educativas virtuais, em tempos de pandemia da Covid-19, desenvolvidas no GGCG da UFSC.

\section{MATERIAIS E MÉTODOS}

Trata-se de um relato de experiência de atividades educativas virtuais de um projeto de extensão universitária, que ocorreu entre os meses de março e julho de 2020. Os encontros e demais atividades do GGCG foram desenvolvidos pelas profissionais que coordenam as atividades do grupo e por duas acadêmicas de Enfermagem da UFSC, bolsistas do projeto de extensão. O GGCG da UFSC, realizado desde 1996, é uma atividade educativa, de extensão, articulada ao ensino e à pesquisa, dirigida a gestantes e acompanhantes. É coordenado por docentes do Departamento de Enfermagem, por psicóloga da Maternidade do Hospital Universitário da UFSC (HU/UFSC), por educadora perinatal e com o apoio de bolsistas acadêmicos do curso de Enfermagem da UFSC.

Diante do cenário de pandemia mundial e a partir da paralisação das atividades presenciais por parte da UFSC, a coordenação do projeto tomou a decisão de manter as atividades dos grupos de número 96 e 97 de forma virtual, sendo que tais encontros ocorreram através de plataforma de webconferência. Os encontros do grupo de número 96 iniciaram-se no dia 12 de março, e a partir do dia 19 desse mesmo mês iniciou-se o processo de transição para atividades virtuais do projeto. Já os encontros do grupo de número 97 ocorreram do dia 14 de maio até o dia 25 de junho. Foram realizados com os dois grupos sete encontros semanais com duração aproximada de duas horas, cujo foco foi trocar experiências e conhecimentos acerca do ciclo gravídico puerperal. O projeto atendeu 47 gestantes e seus acompanhantes.

As participantes do projeto realizaram a inscrição por meio de contato telefônico e preenchimento de formulário online via Google forms ${ }^{\circledR}$. Uma semana antes do início das atividades dos grupos, as bolsistas realizaram contato com todas as inscritas por meio do grupo de aplicativo de mensagem WhatsApp ${ }^{\circledR}$, para confirmar a participação e passar informações sobre o funcionamento da nova modalidade do grupo. Em seguida foram criados dois grupos no WhatsApp ${ }^{\circledR}$, um grupo de conteúdo onde seriam encaminhados textos elaborados pelas coordenadoras, indicações de leituras e vídeos complementares e outro grupo de discussão livre para troca contínua de informações e esclarecimentos de dúvidas entre os participantes, coordenadoras e bolsistas.

Os sete encontros foram divididos entre cinco temas principais: aspectos físicos, emocionais e sociais e culturais da gravidez; preparação e cuidados para o trabalho de parto, parto 
Grupo de gestantes e casais grávidos: educação em saúde em tempos de pandemia

e nascimento; aspectos gerais da amamentação; cuidados físicos e psicológicos no puerpério; e cuidados físicos e psicoafetivos do recém-nascido. Sendo que os conteúdos abordados foram organizados de forma a atender as demandas específicas de cada grupo.

No primeiro encontro, as gestantes e seus acompanhantes elencaram as dúvidas e os temas relacionados ao ciclo gravídico e puerperal que gostariam que fossem debatidos nos encontros semanais. Após este levantamento foi criado um cronograma, descrevendo quais seriam os tópicos de discussão/explicação para cada dia. Este cronograma foi enviado previamente para as participantes via grupo de aplicativo de mensagem WhattsApp ${ }^{\circledR}$.

No segundo encontro o tema discutido foi a gravidez. A partir das demandas trazidas pelos participantes, foram abordados os aspectos emocionais da gestação, papel do pai ou acompanhante, interação com o bebê intraútero, alimentação, exercícios físicos, exames e vacinas essenciais, como lidar com as dores da gravidez e aspectos relacionados à prevenção da Covid-19. Foi um momento muito importante para as mulheres e seus acompanhantes tirarem suas dúvidas sobre os principais cuidados a serem tomados em relação ao distanciamento social.

No terceiro encontro a temática foi sobre trabalho de parto. Nesse dia as gestantes expuseram suas expectativas e receios em relação ao parto, e muitas trouxeram diversas dúvidas em relação ao atendimento das maternidades diante da pandemia. Além disso, foram discutidos os seguintes temas: sinais e sintomas do trabalho de parto, quando ir para a maternidade, fases do trabalho de parto, métodos não-farmacológicos para alívio da dor, indicações de cesárea, plano de parto e violência obstétrica.

No encontro sobre amamentação, como já acontecia nos encontros presenciais, foi convidada para coordenar a discussão uma enfermeira da Central de Incentivo ao Aleitamento Materno do HU/UFSC, que conversou com os participantes sobre livre demanda, pega correta, ordenha, dificuldades com a amamentação, dentre outras dúvidas das gestantes e acompanhantes relacionadas ao referido tema.

O quinto encontro abordou o puerpério, no qual foram discutidos os principais cuidados físicos e emocionais com a mulher durante o pós-parto, relação conjugal x relação parental e o papel da rede de apoio significativa para a família.

No sexto encontro o tema discutido foi cuidado com o recém-nascido, no qual foram abordados tópicos como vacinas, refluxo, sono do bebê, banho do recém-nascido, cuidados psicoafetivos do recém-nascido, além do desenvolvimento psicossocial do bebê.

No último encontro foi apresentado um vídeo das instalações da maternidade do HU/UFSC, com o objetivo de mostrar a área física do Centro Obstétrico e do Alojamento 
Grupo de gestantes e casais grávidos: educação em saúde em tempos de pandemia

Conjunto, e sanar as dúvidas dos participantes em relação a mudanças nas rotinas da maternidade diante da Covid-19.

Além dos encontros online, o contato entre os participantes e as coordenadoras ocorreu concomitantemente por meio dos grupos no aplicativo de mensagem WhatsApp ${ }^{\circledR}$.

Cabe destacar também que, nos encontros presenciais, é oferecido às gestantes e seus acompanhantes prática de exercícios corporais. Nesta nova modalidade, estas orientações foram organizadas na forma de textos e imagens que foram disponibilizadas pelo grupo de mensagem WhatsApp ${ }^{\circledR}$, sendo que a profissional responsável ficou disponível para esclarecer as dúvidas.

\section{RESULTADOS E ANÁLISES}

Participaram dos encontros online do GGCG 47 gestantes. Em relação à faixa etária, uma gestante encontrava-se entre 19 e 25 anos (2\%), vinte e duas entre 26 e 32 anos (47\%), vinte e três entre 33 e 39 anos (49\%), e uma entre 40 e $45 \operatorname{anos}(2 \%)$. No que se refere ao estado civil, nove mulheres são solteiras (19\%), vinte e cinco casadas (53\%), e treze estão em união estável (28\%). Em relação à escolaridade, oito participantes possuem segundo grau completo (17\%), e trinta e nove possuem curso superior (83\%). No que diz respeito aos antecedentes obstétricos, quarenta participantes são primíparas $(85,1 \%)$, cinco possuem um parto anterior $(10 \%)$, uma possui dois partos prévios $(2,1 \%)$ e uma preferiu não responder. Todas realizavam acompanhamento de prénatal, dezessete na rede pública (36\%), vinte e sete na rede privada (57\%), duas nos dois serviços (4\%) e uma não respondeu. Todas as gestantes contaram com a participação de um acompanhante de sua escolha, que, no caso de todas as 47 gestantes, foi o pai do bebê. Em relação à faixa etária, vinte e um encontravam-se entre 26 e 32 anos (45\%), vinte e dois entre 33 e 39 anos (47\%) e quatro entre 40 e 45 anos (9\%).

Destes dados, destaca-se a presença frequente dos acompanhantes nas atividades do grupo. A participação de um acompanhante de escolha da mulher nas consultas de pré-natal e em cursos de preparação para o parto facilita significativamente o seu envolvimento durante a gestação, o processo de nascimento e os cuidados ao recém-nascido e à mulher no puerpério (HOLANDA et al., 2018; JUNGES, BRÜGGEMANN, 2020). Sendo assim, o envolvimento dos acompanhantes nas atividades educativas do GGCG traz inúmeros benefícios à mulher, e em geral resulta em uma experiência de parto e vivência do pós-parto mais satisfatória para todos os envolvidos.

Os grupos de gestantes estimulam a participação das mulheres enquanto sujeito ativo no pré-natal, abrindo um espaço para que elas coloquem seus questionamentos e tirem suas dúvidas, 
Grupo de gestantes e casais grávidos: educação em saúde em tempos de pandemia

podendo ser considerados grupos terapêuticos no que se trata das mudanças emocionais que as mulheres passam durante a gravidez (SILVA et al., 2016).

O distanciamento social imposto pela pandemia trouxe implicações para o acompanhamento de pré-natal, pois algumas consultas foram suspensas até que os serviços de saúde pudessem adequar seu fluxo de atendimento de acordo com as normas técnicas de saúde. A continuidade do projeto de extensão, mesmo de modo remoto, contribuiu para a continuidade da assistência de pré-natal e para que as gestantes e seus acompanhantes tivessem um espaço para esclarecer suas dúvidas, buscar apoio e informações atualizadas sobre a gravidez e o parto, em tempos de pandemia.

Neste contexto, entendendo que o processo de gestação é um período que permeia diversas mudanças físicas, psicológicas e sociais, principalmente frente à pandemia da Covid-19, as atividades de educação em saúde, como os grupos de gestantes, podem ser uma ótima estratégia para promover espaços de troca e compartilhamento de saberes, com a finalidade de empoderar a mulher para viver a gestação, parto e puerpério (TOMASCHEWSKI-BARLEM et al., 2016; MASCARENHAS, 2020).

Um dos objetivos das atividades de extensão é a promoção de iniciativas que expressem o compromisso social das instituições de ensino superior com todas as áreas de educação, tecnologia e produção, cultura, direitos humanos e saúde (BRASIL, 2018). Sendo assim, a interação com a comunidade é um dos pilares essenciais de projetos de extensão, como é o caso do GGCG da UFSC. No contexto da pandemia, esse tipo de atividade se faz essencial, e a criação de um ambiente de educação em saúde proporciona às mulheres e seus acompanhantes acesso a informações de qualidade, com embasamento teórico, e aconselhamento com profissionais da área da saúde, que estão atualizados em relação à situação epidemiológica da Covid-19.

Além disso, cabe ressaltar que a formação cidadã dos estudantes, marcada e constituída pela vivência dos seus conhecimentos de modo multiprofissional e junto a um trabalho interdisciplinar, também é considerada parte essencial das atividades de extensão (BRASIL, 2018). A extensão universitária pode ser uma atividade promotora de interação transformadora mediada por alunos, estimulando o desenvolvimento social e o espírito crítico nos estudantes participantes, assim como contribuindo para a melhoria na qualidade da educação por meio do contato dos extensionistas com realidades concretas na promoção da saúde (FONSECA et al., 2020). Adaptar as atividades do GGCG à modalidade online representou para as discentes uma oportunidade de conhecer novas maneiras de colocar em prática a promoção da saúde, compartilhar conhecimento e trocar experiências, mesmo que a distância. 
Grupo de gestantes e casais grávidos: educação em saúde em tempos de pandemia

As atividades virtuais do GGCG também proporcionaram aos alunos que estavam integrados no projeto de extensão a vivência das estratégias de adaptação da organização dos encontros, assim como acesso a diferentes ferramentas para atividades virtuais, elaboração de novos instrumentos para captação de informações relacionadas aos participantes e, também para avaliação desta nova modalidade de educação em saúde (online).

A promoção da saúde está diretamente ligada à educação em saúde, e é composta de ações voltadas para evitar a exposição da população a fatores de risco para o desenvolvimento de doenças. Sendo assim, para atingir esse objetivo podem ser realizadas atividades de extensão com o objetivo de propagar informações visando ensinar essa comunidade a cuidar da saúde (KRAMERI et al., 2020).

A realização dos encontros possibilitou a formação de uma rede de apoio entre as gestantes e acompanhantes, que continuaram sua interação no grupo de WhastApp ${ }^{\circledR}$ mesmo após a finalização dos encontros do projeto. Contando com o suporte das profissionais coordenadoras e das bolsistas do projeto, as gestantes, puérperas e seus acompanhantes utilizaram o grupo de mensagem para compartilhar experiências, expor e sanar dúvidas, e formar laços de amizade. Esta rede de apoio se mostrou importantíssima neste contexto de distanciamento social, imposto pela pandemia mundial. As bolsistas do projeto monitoraram as mensagens enviadas no grupo de conversas, respondendo questionamentos e fornecendo apoio. Quando necessário, as situações e/ou dúvidas e questionamentos foram encaminhadas às coordenadoras do projeto.

Em geral, o distanciamento social reduz as possibilidades de apoio por parte de familiares e amigos da gestante e/ou casal grávido. Deste modo, o uso de tecnologias virtuais em atividades educativas pode contribuir para a aproximação com familiares, amigos e profissionais da saúde, assim como para a formação de novos laços, amizades, e uma rede de apoio que não depende de interações "cara a cara", como foi o caso das atividades virtuais do GGCG (ALMEIDA; PORTUGAL; ASSIS, 2020). O acesso à informação por meio dos grupos contribui também para o fortalecimento e preparo para lidar com todas as mudanças que o gestar traz para as suas vidas (SILVA et al., 2016).

No século XXI, as tecnologias ou dispositivos móveis reduzem as distâncias físicas e geográficas, propiciando novas formas de relação entre os indivíduos, interações entre pessoas, grupos e espaços, entre aluno e professor, educador e educando e, por que não, entre profissionais de saúde e usuários, não existindo, a priori, nenhum impedimento espacial e/ou temporal (LUCENA; SANTOS; MOTA, 2020). Além disso, contribuem para a formação de comunidades virtuais, ou seja, grupo de pessoas que demonstram interesse em partilhar práticas comuns e 
Grupo de gestantes e casais grávidos: educação em saúde em tempos de pandemia

estabelecem uma discussão pública com duração e intensidade suficientes para criar redes de relações pessoais no ciberespaço (NUNES; VIEIRA; MOREIRA, 2020). Além disso, essas comunidades criam um espaço de informações e conexões que contribuem para aumentar a percepção de si e dos outros, compreender melhor a realidade do mundo, construir novos conhecimentos (NORONHA; XAVIER; OLIVEIRA, 2020) e aumentar a autonomia.

A estratégia de ofertar grupo de gestantes online possibilita novas formas de narrar as experiências e vivências sociais, culturais e políticas, bem como de ressignificar as possibilidades de consumir e produzir informações, as quais contribuem para novas formas de aprendizagem (LUCENA; SANTOS; MOTA, 2020). Espera-se que a experiência do GGCG, relatada neste texto, estimule os profissionais de saúde a desenvolverem, no seu processo de trabalho, atividades educativas grupais online com e para as gestantes e seus acompanhantes.

\section{CONSIDERAÇÕES FINAIS}

O desenvolvimento do projeto GGCG de maneira online proporcionou troca de experiências e construção do conhecimento, já que as estratégias educacionais possuem um grande impacto na vivência da gestação. Os encontros virtuais do grupo serviram como um local de troca de saberes e favoreceram a formação de uma rede de apoio que se prolonga após o nascimento dos bebês.

As atividades educativas realizadas com as gestantes e seus acompanhantes vão ao encontro dos objetivos da extensão universitária, pois promovem ações que geram impactos na comunidade, preparando a mulher e seu acompanhante para a vivência da gravidez, parto e nascimento em tempos de pandemia. O desenvolvimento das atividades online do GGCG da UFSC criou um espaço de reflexão sobre diversos aspectos do ciclo gravídico-puerperal, bem como possibilitou maior preparo para a vivência da maternidade e paternidade em tempos de pandemia.

Destaca-se que a adaptação do projeto para o formato online foi positiva para os docentes, profissionais, discentes e comunidade envolvida, pois aproximou a universidade da população em um contexto em que as interações humanas presenciais estão limitadas. Inovar a extensão universitária é necessário diante do desafio imposto pela pandemia da Covid-19. Devido à procura constante de gestantes e acompanhantes para a participação no GGCG da UFSC, além da avaliação positiva desta recente modalidade online, e sobretudo para manutenção da contribuição do projeto à comunidade, serão mantidas as atividades do GGCG de maneira virtual enquanto forem recomendadas as medidas de distanciamento social devido à pandemia da Covid-19. 
Grupo de gestantes e casais grávidos: educação em saúde em tempos de pandemia

\section{REFERÊNCIAS}

ALMEIDA, M.O.; PORTUGAL, T.M.; ASSIS, T. J.C.F. Gestantes e COVID-19: isolamento como fator de impacto físico e psíquico. Rev. Bras. Saude Mater. Infant., Recife, v. 20, n. 2, p. 599-602, jun. 2020. Doi: https://doi.org/10.1590/1806-93042020000200015

AQUINO, Estela M. L. et al . Medidas de distanciamento social no controle da pandemia de COVID-19: potenciais impactos e desafios no Brasil. Ciênc. saúde coletiva, Rio de Janeiro, v. 25, supl. 1, p. 2423-2446, June 2020 . Available from

http://www.scielo.br/scielo.php?script=sci_arttext\&pid=S1413-

$81232020006702423 \& \operatorname{lng}=$ en\&nrm=iso. access on 12 Apr. 2021. Epub June 05, 2020.

https://doi.org/10.1590/1413-81232020256.1.10502020.

ARAÚJO, J.L.; OLIVEIRA, K.K.D; FREITAS, R.J.M. Organização da assistência hospitalar de referência para COVID-19: Relato de experiência. Brazilian Journal Of Development, Curitiba, v.6, n.5, p. 29326-29339. mai. 2020.

Disponível:https://www.brazilianjournals.com/index.php/BRJD/article/view/10334/8648.

Acesso em 12 abr.2021.

BRASIL. Ministério da Educação. Estabelece as Diretrizes para a Extensão na Educação Superior Brasileira e regulamenta o disposto na Meta 12.7 da Lei no 13.005/201. Resolução n. 7, de 07 de dezembro de 2018. Brasília, 2018. Disponível em:

http:/ /portal.mec.gov.br/index.php?option $=$ com_docman\&view $=$ download\&alias $=104251$ rces007-18\&category_slug=dezembro-2018-pdf\&Itemid=30192. Acesso em: 13 de agosto de 2020.

ESTRELA, F.M. et al. Gestantes no contexto da pandemia da Covid-19: reflexões e desafios.

Physis, Rio de Janeiro, v. 30, n.2. jul 2020. Disponível em:

https://www.scielo.br/scielo.php?pid=S0103-73312020000200314\&script=sci_arttext\&tlng=pt.

Acesso em 20 de julho de 2020.

FONSECA, Y.S. et al. Ação extensionista de promoção da saúde para estudantes universitários.

Extensio: R. Eletr. de Extensão, v. 17 n. 35, p. 81-95, 2020. Disponível em:

https://periodicos.ufsc.br/index.php/extensio/article/view/1807-0221.2020v17n35p81/43029.

Acesso em: 17 de agosto de 2020.

GONÇALVES, C.B. Educação a distância: a aprender online. Rev. ARETÉ, Manaus, v. 4, n. 6. p. 24-34, jul 2011. Disponível em:

http://periodicos.uea.edu.br/index.php/arete/article/view/12/6. Acesso em: 13 de agosto de 2020.

HOLANDA, S.M. et al. Influência da participação do companheiro no pré-natal: satisfação de primíparas quanto ao apoio no parto. Texto contexto - enferm., Florianópolis. v. 27, n. 2 , e3800016 2018. Disponível em: https:/ /www.scielo.br/scielo.php?pid=S0104-

07072018000200317\&script=sci_arttext\&tlng=pt. Acesso em: 18 de agosto de 2020.

JUNGES, C. F.; BRUGGEMANN, O. M. Fatores associados ao apoio realizado à mulher durante o parto pelos acompanhantes em maternidades públicas. Texto contexto - enferm., 
Grupo de gestantes e casais grávidos: educação em saúde em tempos de pandemia

Florianópolis, v. 29, e20180239, 2020. Disponível em: https://doi.org/10.1590/1980-265x-tce2018-0239. Acesso em 12 abr. 2021.

KRAMERI, D.G. et al. Extensão Universitária e ações de educação em saúde para prevenção ao COVID-19. Anuário Pesquisa e Extensão UNOESC, Joaçaba. v.5, jun 2020. Disponível em: https://unoesc.emnuvens.com.br/apeuj/article/view/24329/14280. Acesso em: 17 de agosto de 2020.

LUCENA, S.; SANTOS, S. V. C. de A.; MOTA, G. da M. da. Formação continuada de professores com as tecnologias móveis digitais. Revista Educação em Foco, v. 25, n. 1, p. 232248, 2020. Disponível em: https://periodicos.ufff.br/index.php/edufoco/article/view/30440. Acesso em 15 agosto de 2020.

MASCARENHAS, V. H.A. et al. COVID-19 e a produção de conhecimento sobre as recomendações na gravidez: revisão de escopo. Rev. Latino-Am. Enfermagem, Ribeirão Preto. v. 28, e3348, 2020. Disponível em:

https://www.scielo.br/scielo.php?script=sci_arttext\&pid=S0104-

11692020000100606\&lng=pt\&nrm=iso\&tlng=pt. Acesso em: 18 de agosto de 2020.

NUNES, G.P. Grupo de Gestantes como ferramenta de instrumentalização e potencialização do cuidado. Revista de Extensão e Cultura, Florianópolis, v. 1, n. 1, p.1-16, out. 2017.

SILVA, E. P. et al. Avaliação do pré-natal na atenção primária no Nordeste do Brasil: fatores associados à sua adequação. Rev Saúde Pública, São Paulo, v. 53, n.43, p. 43-53, 2019.

Disponível em:https://doi.org/10.11606/s1518-8787.2019053001024

Acesso em 20 de julho de 2020.

TOMASCHEWSKI-BARLEM, J. G. et al. Promovendo a autoestima na gestação: foco no acolhimento. Enferm. Foco. v. 7, n. 2, p. 83-86, 2016. Disponível em:

http://revista.cofen.gov.br/index.php/enfermagem/article/view/801/326. Acesso em: 18 de agosto de 2020 .

UNIVERSIDADE FEDERAL DE SANTA CATARINA [homepage da internet]. Notícias da UFSC. Administração Central; 13 de março de 2020. Disponível em: https://noticias.ufsc.br/2020/03/administracao-central-anuncia-medidas-de-contingencia-naufsc-em-razao-da-pandemia-de-covid-19/. Acesso em: 14 de abril de 2021.

Recebido em: 31/08/2020

Aceito em: 07/07/2021 